\title{
Editorial
}

\section{Mathematical and Computational Topics in Design Studies}

\author{
Teen-Hang Meen, ${ }^{1}$ Stephen D. Prior, ${ }^{2}$ Kuei-Shu Hsu, ${ }^{3}$ and Artde Donald Kin-Tak Lam ${ }^{4}$ \\ ${ }^{1}$ Department of Electronic Engineering, National Formosa University, Yunlin 632, Taiwan \\ ${ }^{2}$ Aeronautics, Astronautics and Computational Engineering, University of Southampton, Southampton SO17 1BJ, UK \\ ${ }^{3}$ Department of Applied Geoinformatics, Chia Nan University of Pharmacy \& Science, Tainan City 717, Taiwan \\ ${ }^{4}$ College of Physics and Information Engineering, Fuzhou University, Fuzhou, Fujian 350116, China
}

Correspondence should be addressed to Teen-Hang Meen; thmeen@nfu.edu.tw

Received 23 March 2015; Accepted 23 March 2015

Copyright (C) 2015 Teen-Hang Meen et al. This is an open access article distributed under the Creative Commons Attribution License, which permits unrestricted use, distribution, and reproduction in any medium, provided the original work is properly cited.

For increasing the added value and competitiveness of product, recently, design studies and their applications have been paid closer attention than ever. Good design has always been considered as the perfect combination of creativity and experience of the designer. In contrast to engineering, design problems had been thought as unsolvable by scientific methods. Now, due to the advances of design studies, many new design methods have been proposed. These methods reduce the time consumption of design by introducing some technical procedures and rules. Particularly, the popularization of computer and the release of many powerful CAD tools have greatly shortened the design cycle. With simulation software, designers reduce the time and cost of prototyping. The advancement of machine learning, data mining, and other techniques provides excellent tools for information collecting, data analyzing, behavior forecast, and decision-making. Therefore, the design complexity of consumer behavior, social and cultural preferences, environmental impacts, and economic factors can be taken into account properly.

Design studies include not only the study of design itself but also marketing, service, and strategy of design. In addition, qualitative and quantitative thinking of its influence and the study of design education are all involved. The researches of design have been improved enormously by introducing the quantitative methodologies of engineering and social science.

This special issue selects papers about the topics of (1) various computational topics of methodologies and procedures of design, (2) mathematical techniques to reduce time and cost of design, (3) mathematical tools or methods that assist in the marketing or decision-making of design, (4) computational issues to promote design education, (5) computational topics about design services or cultural, environmental, social, and economic aspects of design, and (6) mathematical modeling and computation about design procedure, strategy, and management. This special issue enables interdisciplinary collaboration between design and engineering technologists in the academic and industrial fields.

Teen-Hang Meen Stephen D. Prior

Kuei-Shu Hsu Artde Donald Kin-Tak Lam 


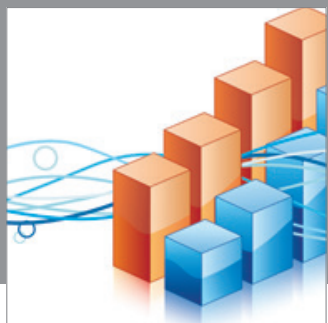

Advances in

Operations Research

mansans

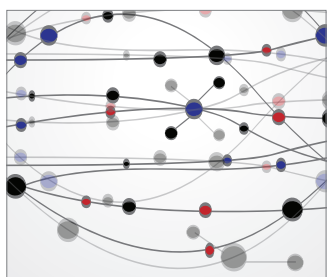

The Scientific World Journal
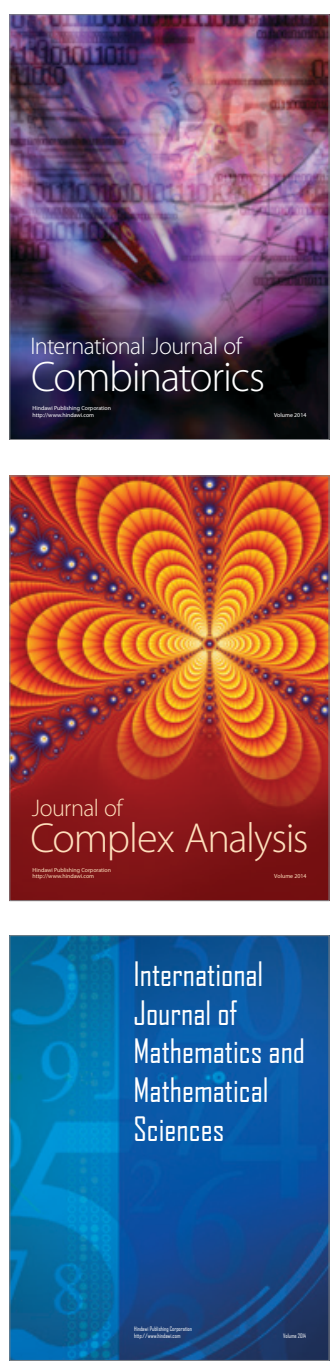
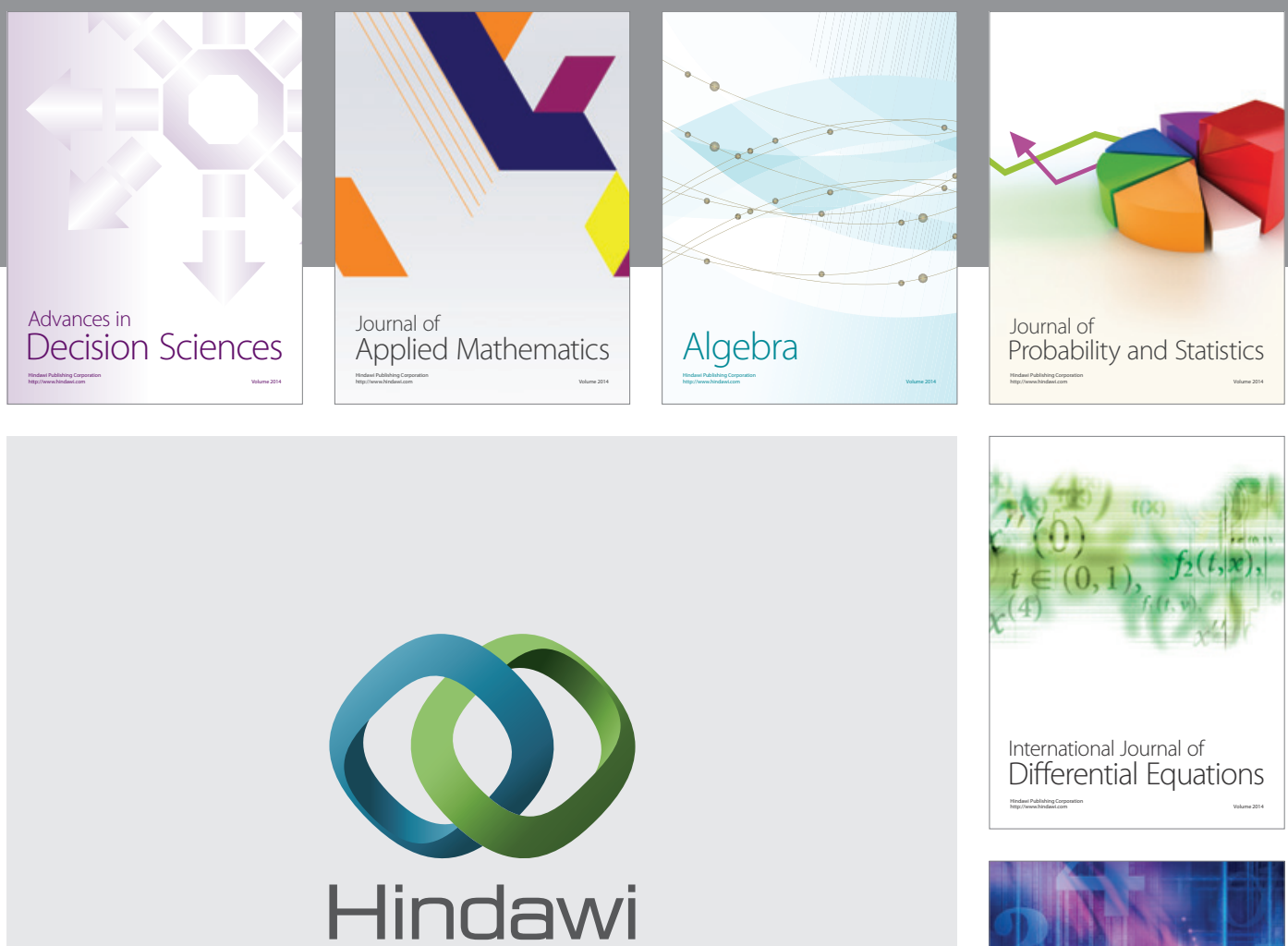

Submit your manuscripts at http://www.hindawi.com
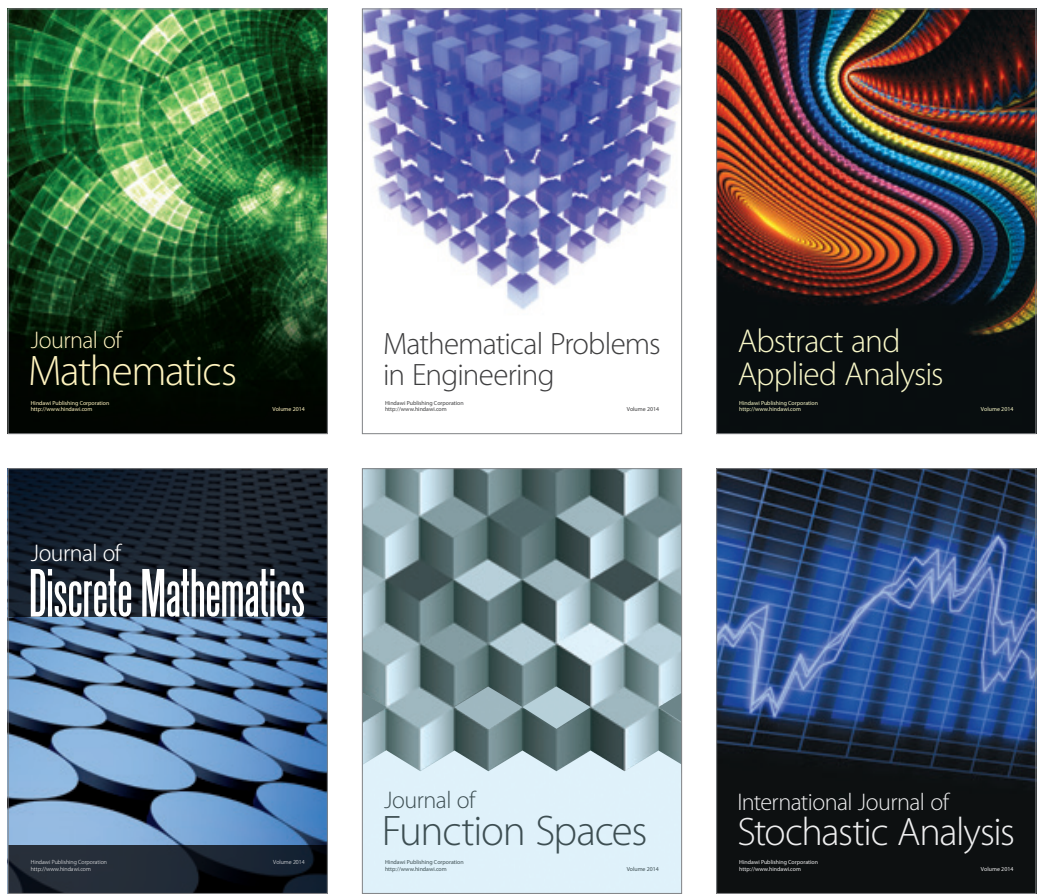

Journal of

Function Spaces

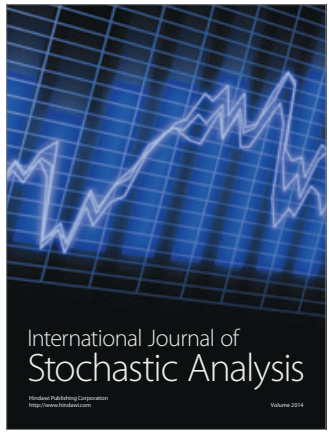

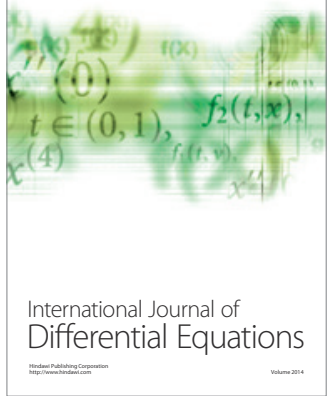
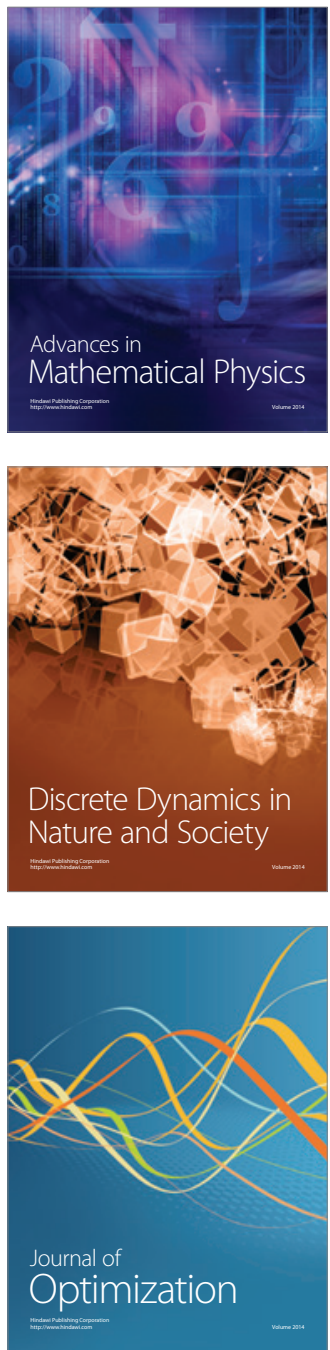